\title{
PREVALENCE OF OCULAR SURFACE DISEASE IN GLAUCOMA PATIENTS USING ANTI-GLAUCOMA MEDICATONS.
}

Vinutha B V¹, N. V. V. Himamshu², Sundeep³, Niveditha H4, Pooja Patil5, Liji P6, M. S. Smitha Gowda7.

1. Senior Resident, Department of Ophthalmology, Kempegowda Institute of Medical Sciences and Research Center.

2. Professor and HOD, Department of Ophthalmology, Kempegowda Institute of Medical Sciences and Research Center.

3. Associate Professor, Department of Ophthalmology, Kempegowda Institute of Medical Sciences and Research Center.

4. Associate Professor, Department of Ophthalmology, Kempegowda Institute of Medical Sciences and Research Center.

5. Post Graduate, Department of Ophthalmology, Kempegowda Institute of Medical Sciences and Research Center.

6. Post Graduate Post Graduate, Department of Ophthalmology, Kempegowda Institute of Medical Sciences and Research Center.

\section{CORRESPONDING AUTHOR}

Dr. Vinutha B. V,

\# 012, shuddha shelters,

Behind shantiniketan school,

Someshwara layout,

Bilekahalli, Bangalore - 76 .

Email- vinutha_seetharam@yahoo.co.in

\section{HOW TO CITE THIS ARTICLE:}

Vinutha B V, N. V. V. Himamshu, Sundeep, Niveditha H, Pooja Patil, Liji P, M. S. Smitha Gowda."Prevalence of Ocular Surface Disease in Glaucoma Patients using Anti-Glaucoma Medications". Journal of Evolution of Medical and Dental Sciences 2013; Vol2, Issue 24, June 17; Page: 4308-4314.

ABSTRACT: AIM; To evaluate the prevalence of ocular surface disorder (OSD) in patients using anti-glaucoma medications METHODS; 150 eyes of 75 glaucoma patients on topical anti-glaucoma medication with preservatives were studied. Patients with history of use of topical corticosteroids, cyclosporine, anti-inflammatory drugs or other topical medications within last 3 months were excluded. Patients completed Ocular Surface Disease Index questionnaire, underwent evaluation by Schirmer test, tear breakup time, corneal and conjunctival lissamine green staining. RESULTS; Mean age of patients was 56.5 (range 23 - 80) years. 50 (67\%) patients were males and 25 (33\%) patients were females.56\% patients had primary open angle glaucoma. $41 \%$ patients had primary angle glaucoma. 3\% patients had ocular hypertension. $44(59 \%)$ patients were on single medication. 25 (33\%) patients were on two medications. $6(8 \%)$ patients were on three medications. Mean duration of using medication was 3.8 yrs (range; 5 months - 16 yrs). Duration less than $1 \mathrm{yrs}-10(13 \%)$ patients. 1 to $5 \mathrm{yrs}-36(48 \%)$ patients. 5 to $1 \mathrm{yrs}-19(26 \%)$ patients. More than $10 y$ rs $-10(13 \%)$ patients. $32 \%$ patients reported symptoms in at least one eye. Severe symptoms were reported by none. Tear breakup time was abnormal in $54.5 \%$ patients, severe abnormality in $20.5 \%$ patients. Schirmer testing showed $50.5 \%$ patients with decreased tear production in at least one eye, severe tear deficiency in $21.5 \%$ patients. Lissamine green staining of conjunctival and cornea showed positive results in $47 \%$ patients. None had severe staining. As 
duration of treatment and number of medications increased, more patients became symptomatic and test results became abnormal. CONCLUSION; High prevalence of ocular surface diseases was noted in patients using anti-glaucoma medications, higher the incidence with increasing number of medications and longer duration of usage. Ocular surface disease must be kept in mind in symptomatic patients as it is likely to affect drug compliance

KEY WORDS; anti-glaucoma agents, ocular surface disease, benzalkonium chloride, dry eye

INTRODUCTION: Glaucoma is one of the leading causes of blindness in the world today. Majority of glaucoma patients receive topical medical treatment in the form of eye drops. Glaucoma is progressive disease requiring long term treatment often rest of the life. Some of these patients require multiple medications. Anti-glaucoma medications are generally well tolerated. Preservatives are used in these medications to maintain sterility. Long term use of these preserved anti-glaucoma medications is shown to adversely affect conjunctiva and cornea, leading to increased risk of developing or worsening of ocular surface disease ${ }^{1}$.

OSD is characterized by inadequate quantity of tear, impairment of protective tear film and ocular surface damage. OSD symptoms include irritation, burning, foreign body sensation, dryness, photophobia, fatigue and fluctuating visual acuity. Several factors such as age, race, sex, associated diabetes mellitus and meibomian gland dysfunction are considered to influence the prevalence of $\mathrm{OSD}^{2}$. OSD and glaucoma are both prevalent in elderly, often present as comorbid conditions.

METHODS: This is a prospective observational study. 75 patients attending glaucoma clinic of Kempegowda Medical college Ophthalmology department were studied. Patients aged 18 years and above with Primary Open Angle Glaucoma, primary angle closure glaucoma and ocular hypertension were recruited. These patients were being treated with topical anti-glaucoma medications with preservatives. Patients with history of using any other topical medications, YAG laser, any anterior segment pathology or ocular surgery were excluded.

Informed consent was taken from selected patients. Demographic information, brief medical history and information on concomitant medicine use were obtained from patient's medical records. All the eligible patients were asked to complete Ocular Surface Disease Index [OSDI] questionnaire. After completing the OSDI questionnaire, patients under went three standard clinical tests for the detection of ocular surface disorder - Schirmer 1 test, Tear breakup time (TBUT) and Lissamine green staining of conjunctiva and cornea.

The OSDI questionnaire was designed as a screening survey to assess symptoms and their impact on vision related function ${ }^{3}$. The12 questions of OSDI questionnaire were graded on the scale of 0 to 4. 0 - none of the time, 1 - some of the time, 2 - half of the time, 3 - most of the time, 4 - all the time. The total OSDI score was calculated using the formula

OSDI = sum of scores for all questions asked X 25 Total number of questions answered

Using OSDI score, patients were categorized as normal (score 0 - 12), mild OSD symptoms (score13 - 22), moderate symptoms (score 23 - 32) and severe symptoms (33-100).

Schirmer test 1 (without anesthesia) was done using Whatmann no.41 filter paper (5X35 $\mathrm{mm}$ ). the filter paper strip was folded over at the labeled end and hocked in the lower cul de sac over the junction of outer and middle one third of lower lid while patient is looking slightly upwards. The patients were allowed to blink normally. After 5 minutes, filter paper strip was 
removed. Moistened filter paper section from the fold was measured in millimeters immediately. If the filter paper strip was completely wet before the time elapsed, it was removed immediately and the time necessary for this was measured. Schirmer test value above $10 \mathrm{~mm}$ was considered normal, 6to $10 \mathrm{~mm}$ mild to moderate and less than, $5 \mathrm{~mm}$ severe tear deficiency.

Lissamine green staining of conjunctiva and cornea was done by inserting one drop of lissamine green into lower conjunctival sac. Corneal and conjunctival staining was evaluated using moderate light intensity after 30 seconds but before 2 minute had elapsed after installation. Staining was graded using Oxford Scheme. The severity was graded as $0-1$ normal, 2-3 mild to moderate and 4-5 severe.

Tear Break Up Time (TBUT) -2\% sodium fluorescein dye was administered into inferior culde sac. Patient was asked to blink several times so that fluorescein dye distributes itself uniformly. Examination was performed with slit lamp using cobalt blue illumination. Patient was asked to blink and keep open without further blinking. TBUT was measured over the cornea as the time from last blink until the appearance of first black spot in the green yellow fluorescein. TBUT results were graded as more than $\geq 10$ seconds - normal, 5 - 9 seconds - mild to moderate, «5seconds - severe.

RESULTS: Seventy five patients (150 eyes) participated and completed all the tests involved in the study. 50 patients $(67 \%)$ were males and 25 patients(33\%) were females, mean age of the patients was 56.9 years (range 22- 88years). 40patients (56\%) were diagnosed with primary open angle glaucoma, 30 patients (41\%) with primary angle closure glaucoma and 5 patients (3\%) with ocular hypertension. Mean duration of using medication was 3.8years (range 5 months to 16 years). 10 patients (13\%) were using medication for less than 1 year, 36 patients (48\%) between 1 - 5 years, 19 patients (26\%) between 5-10 years and 10 patients (13\%) more than 10 years. 44 patients $(58.6 \%)$ were on single medication, 25 patients $(33.3 \%)$ were on two medications and 6 patients $(8 \%)$ were on three medications.

Results obtained from OSI questionnaire and three tests are summarized in table 1 and graph1. Based on OSDI questionnaire, $32 \%$ patients reported symptoms in at least one eye. Severe symptoms were reported by none. Tear breakup time was abnormal in $54.5 \%$ patients, severe abnormality in $20.5 \%$ patients. Schirmer test showed patients $50.5 \%$ with mild to moderate decrease in tear film production in at least on eye, severe tear deficiency in patients21.5\%.Lissamine green staining of conjunctiva and cornea showed mild to moderate staining in patients $46.5 \%$, none having severe staining.

Table 2 and graphs 2A, 2B, 2C, 2D shows relationship between OSDI and three test results with respect to duration of treatment. In patients using topical medication less than one year, none of the patients were symptomatic. As the duration of treatment increased, more patients became symptomatic, with $66 \%$ showing mild to moderate symptoms. Schirmer test results also showed that in patients using treatment less than one year, $60 \%$ had normal, $30 \%$ mild to moderate decrease in tear film production in at least on eye and severe tear deficiency in $10 \%$ patients. In patients using treatment more than 10 years, only $20 \%$ had normal tear production, $60 \%$ mild to moderate decrease in tear film production and $20 \%$ severe tear deficiency. TBUT value was normal in $40 \%$, mild - moderate in $40 \%$ and severe in $20 \%$ in patients using treatment less than one year. Patients using treatment more than ten years, TBUT was normal only in 7\%, mild - 
moderate in $80 \%$. Lissamine green staining of conjunctiva and cornea was normal in $90 \%$ and $10 \%$ showed mild to moderate staining in patients using medication less than one year. Whereas in patients using medication more than ten years, only $20 \%$ showed normal staining and $80 \%$ mild to moderate staining.

Table 3 shows OSDI score and test results with respect to number of medications used. As the number of medications increased, OSDI score and test results became more abnormal.

DISCUSSION: Ocular surface disease and glaucoma are both prevalent in old age. OSD prevalence increases with age, affecting approximately 11\% in 40-59 age group and 18\% in older than 80 years in general population ${ }^{4,5}$. Prevalence of primary open angle glaucoma increases with age, affecting approximately $1 \%$ in $40-49$ age group and $8 \%$ in older than 80 years $^{6}$. The German Glaucoma and Dry Eye Register showed that incidence of dry eye increases with age, more in women, prevalence increases because of long term use of multiple anti-glaucoma medications ${ }^{7}$.

Robert D Fuechtner et al study reported that $48.4 \%$ patients had OSD symptoms, $21.3 \%$ mild, $13.3 \%$ moderate and $13.8 \%$ severe OSD scores ${ }^{7}$.

Leung et al in their study reported 59\% patients had symptoms of dry eye and severe symptoms in $27 \%$. They also noted that use of more benzalkonium chloride containing eye drop was significantly associated with higher prevalence of abnormal results on lissamine green stain ${ }^{9}$.

In our study, OSD index score was less compared to other studies, none having severe symptoms. In our study Schirmer test, T-BUT values and lissamine green staining correlated with these studies.

Benzalkonium chloride is most commonly used preservative. it is a quaternary ammonium compound made up of a mixture alkylbenzyldimethylammonium chloride homologues. The concentration of benzalkonium chloride used in ophthalmic eye drops ranges at $0.004 \%$ $0.025 \%$.Evidence from animal and cell line studies has shown that benzalkonium chloride is directly toxic to ocular tissues ${ }^{10,11}$. It exerts detergent effect on lipid layer of tear film, reducing its stability causing it to evaporate more rapidly and results in increased ocular dryness ${ }^{12}$. Benzalkonium chloride has destructive effect on mucosal glands, decreasing goblet cells and production of protective mucus layer ${ }^{13}$.Benzalkoniumchloride causes inflammation of conjunctival layer leading to subconjunctival fibrosis. Subconjunctival fibrosis develops because of increased fibroblastic density in subepithelial substantia propria, linked to an increase in inflammatory cells ${ }^{14,15^{\prime 16}}$. Immunohistochemical study of conjunctival and trabecular specimens from surgical patients receiving preserved anti-glaucoma treatment revealed significantly greater expression of fibroblastic and inflammatory markers compared with those who did not receive topical treatment ${ }^{16}$. Expression of fibroblastic and inflammatory markers was higher in patients receiving multiple drops compared to those using single medication ${ }^{17}$. Long term use of topical anti-glaucoma therapy, particularly combination treatment regimes has been associated with failure of glaucoma filtration surgery ${ }^{16}$.The toxic effects of preservatives are initially subclinical. Long term cumulative application of preservatives containing anti-glaucoma medications to the ocular surface is associated with development of signs and symptoms of ocular discomfort. Studies have shown that significant higher proportion of patients receiving preserved medications experience discomfort or pain during therapy compared with those treated with preservative free $\operatorname{drugs}^{18}$. 
Our study had a limitation. We did not have control group that could have helped to provide further insight into the relationship between use of eye drops with and without preservatives and OSD.

Conclusion; High prevalence of ocular surface diseases was noted in patients using anti-glaucoma medications, higher the incidence with increasing number of medications and longer duration of usage. Preservative agents have dose dependent toxic effects, compromising tear film stability leading to conjunctival and corneal damage. Patients with side effects are less likely to comply with their treatment and compromising quality of life. Ocular surface disease must be kept in mind in symptomatic patients as it is likely to affect drug compliance.

\section{BIBLIOGRAPHY;}

1. Pisella PJ, Pouliquen P, Baudouin C. Prevalence of ocular symptoms and signs with preserved and preservative free glaucoma medication. Br J Ophthalmol.2002;86: 418-423

2. Brewitt H, Sistani F. Dry eye disease; the scale of the problem. Surv Ophthalmol.2001; 45(suppl2): S199-202

3. Schiffman RM, Christianson MD, Jacobsen G, et al. reliability and validity of the ocular surface disease index. Arch Ophthalmol.2000;118:615-621

4. Moss SE, Klein R, Elein BE. Prevalence and risk factors for dry eye syndrom. Arch Ophthalmol.2000;118:1264-1268

5. Schein OD, Munoz B, Tielsch JM, et al. prevalence of dry eye among the elderly. Am J Ophthalmol.1997;124

6. Friedman DS, Wolfs RC, et al. prevalence of open angle glaucoma among adults in the United States. Arch Ophthalmol.2004; 122:532-538

7. Christophe Baudouin. The ocular surface in glaucoma. J Cornea.2009;28: S14-S19

8. Robert D Fechtner, Davidg. Gogfrey et al. Prevalence of ocular surface complaints in patients with glaucoma using topical intraocular pressure lowering medications. J Cornea.2010;29:618-621

9. Leung Ew, Medeiros FA, Weinreb RN. Prevalence of ocular surface disease in glaucoma patients.J glaucoma.2008;17:350-355

10. Wilson WS, Duncan AJ, Jay JL. Effect of benzalkonium chloride on the stability of the precorneal tear film in rabbit and man. Br J Ophthalmol.1975;59:667-669

11. Kuppens EV, de Jong CA, Stolwiijk TR, et al. effect of timolol with and without preservatives on the basal tear turn over in glaucoma. Br J Ophthalmol.1995;79:339-342

12. Herresras JM, Pastor JC, Calong M, et al. Ophthalmology. 1992;99:1082-1088

13. Baudouin C, Pisella PJ, Fallacer K, et al. ocular surface inflammatory changes induced by topical anti-glaucoma drugs; human and animal studies. Ophthalmology. 1999;106:556563

14. Sherwood MB, Grierson I, Millar L, et al. long term morphological effects of topical antiglaucoma drugs on the conjunctiva and tenon's capsule in glaucoma patients. Ophthalmology.1989;96:327-335

15. Broadway DC, Grieson I, O'Brien C, et al. Adverse effects of topical anti-glaucoma medication l. The conjunctival cell profile. Arch Ophthalmol.1994;112:1437-1445 
16. Broadway DC, Grieson I, O’Brien C, et al. Adverse effects of topical antiglaucoma medication ll. The outcome of filtration surgery. Arch Ophthalmol.1994;112:1446-1454

17. Noecker RJ, Herrygers LA, Anwaruddin R. corneal and conjunctival changes caused by commonly used glaucoma medications. J Cornea. 2004;23:490-496

18. Jaenen N, Baudouin C, Pouliquen P, et al. Ocular symptoms and signs with preserved and preservative free glaucoma medications. Eur J Ophthamol.2007;17:341-349

TABLE 1

\begin{tabular}{|l|l|l|l|l|}
\hline Results & OSD (\%) & Schirmer (\%) & T BUT (\%) & Lissamine green \\
\hline Normal & 68 & 27 & 26 & 53 \\
& & & & \\
\hline Mild-moderate & 32 & 51 & 54 & 47 \\
\hline Severe & 0 & 35 & 20 & 0 \\
\hline
\end{tabular}

TABLE 2

\begin{tabular}{|c|c|c|c|c|c|c|c|c|c|c|c|c|c|}
\hline \multirow{2}{*}{$\begin{array}{l}\text { Duration } \\
\text { of } \\
\text { treatment }\end{array}$} & \multirow{2}{*}{$\begin{array}{l}\text { No. of } \\
\text { patients }\end{array}$} & \multicolumn{3}{|c|}{ OSD (\%) } & \multicolumn{3}{|c|}{ Schirmer (\%) } & \multicolumn{3}{|c|}{ T BUT (\%) } & \multicolumn{3}{|c|}{ Lisamine green (\%) } \\
\hline & & normal & $\begin{array}{l}\text { mild- } \\
\text { mod }\end{array}$ & severe & normal & $\begin{array}{l}\text { mild- } \\
\text { mod }\end{array}$ & severe & normal & $\begin{array}{l}\text { mild- } \\
\text { mod }\end{array}$ & severe & normal & $\begin{array}{l}\text { Mild- } \\
\text { mod }\end{array}$ & severe \\
\hline ?1yr & 10 & 100 & 0 & 0 & 60 & 30 & 10 & 40 & 40 & 20 & 90 & 10 & 0 \\
\hline $1-5 y r$ & 36 & 78 & 22 & 0 & 59 & 30 & 11 & 39 & 41 & 20 & 69 & 31 & 0 \\
\hline $5-10 y r$ & 19 & 65 & 35 & 0 & 40 & 47 & 13 & 13 & 70 & 17 & 42 & 58 & 0 \\
\hline ? $10 \mathrm{yr}$ & 10 & 44 & 66 & 0 & 20 & 60 & 20 & 7 & 83 & 10 & 20 & 80 & 0 \\
\hline
\end{tabular}

TABLE 3

\begin{tabular}{|c|c|c|c|c|c|c|c|c|c|c|c|c|c|}
\hline \multirow{2}{*}{$\begin{array}{l}\text { No } \\
\text { of } \\
\text { dru } \\
\text { gs }\end{array}$} & \multirow{2}{*}{$\begin{array}{l}\text { No. of } \\
\text { patie } \\
\text { nts }\end{array}$} & \multicolumn{3}{|c|}{ OSD (\%) } & \multicolumn{3}{|c|}{ Schirmer (\%) } & \multicolumn{3}{|c|}{ T BUT (\%) } & \multicolumn{3}{|c|}{$\begin{array}{l}\text { Lisamine } \\
(\%)\end{array}$} \\
\hline & & $\begin{array}{l}\text { norm } \\
\text { al }\end{array}$ & $\begin{array}{l}\text { Mil } \\
\text { d- } \\
\text { mo } \\
\text { d }\end{array}$ & $\begin{array}{l}\text { seve } \\
\text { re }\end{array}$ & $\begin{array}{l}\text { norm } \\
\text { al }\end{array}$ & $\begin{array}{l}\text { Mil } \\
\text { d- } \\
\text { mo } \\
\text { d }\end{array}$ & $\begin{array}{l}\text { seve } \\
\text { re }\end{array}$ & $\begin{array}{l}\text { norm } \\
\text { al }\end{array}$ & $\begin{array}{l}\text { Mil } \\
\text { d- } \\
\text { mo } \\
\text { d }\end{array}$ & $\begin{array}{l}\text { seve } \\
\text { re }\end{array}$ & $\begin{array}{l}\text { norm } \\
\text { al }\end{array}$ & $\begin{array}{l}\text { Mil } \\
\text { d- } \\
\text { mo } \\
\text { d }\end{array}$ & $\begin{array}{l}\text { seve } \\
\text { re }\end{array}$ \\
\hline 1 & 59 & 78 & 22 & 0 & 60 & 10 & 30 & 43 & 43 & 14 & 81 & 19 & 0 \\
\hline 2 & 33 & 76 & 24 & 0 & 58 & 25 & 17 & 32 & 50 & 33 & 71 & 29 & 0 \\
\hline 3 & 8 & 71 & 29 & 0 & 57 & 29 & 14 & 17 & 50 & 33 & 70 & 30 & 0 \\
\hline
\end{tabular}




\section{ORIGINAL ARTICLE}

Graph 1

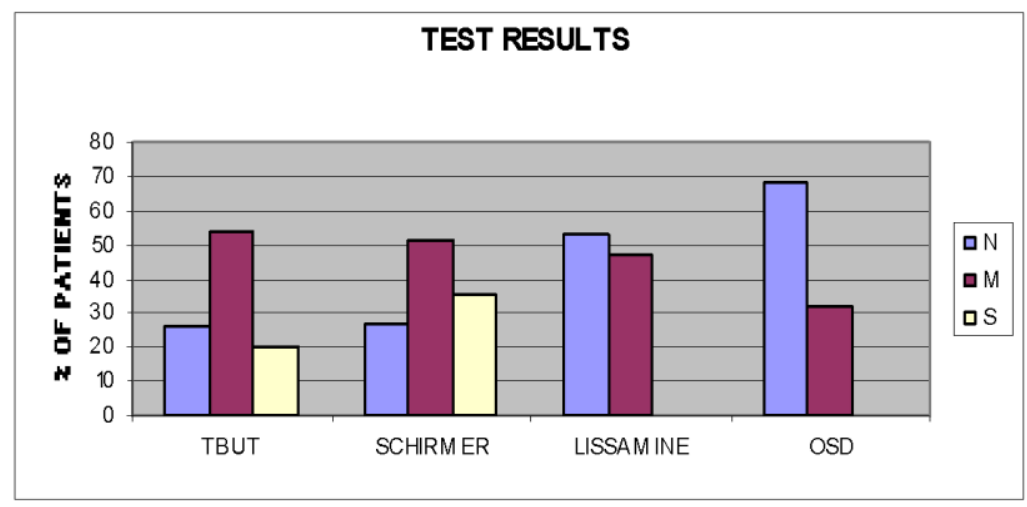

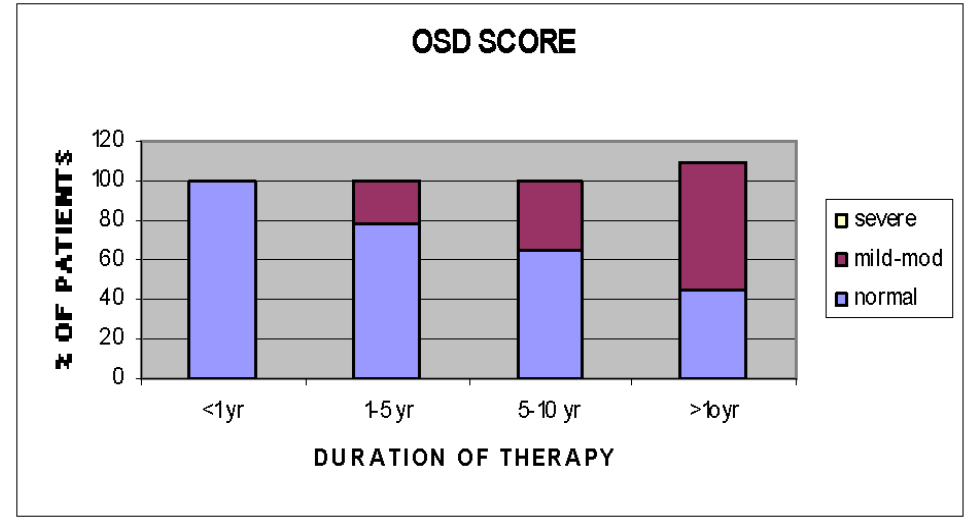

$2 \mathrm{~A}$

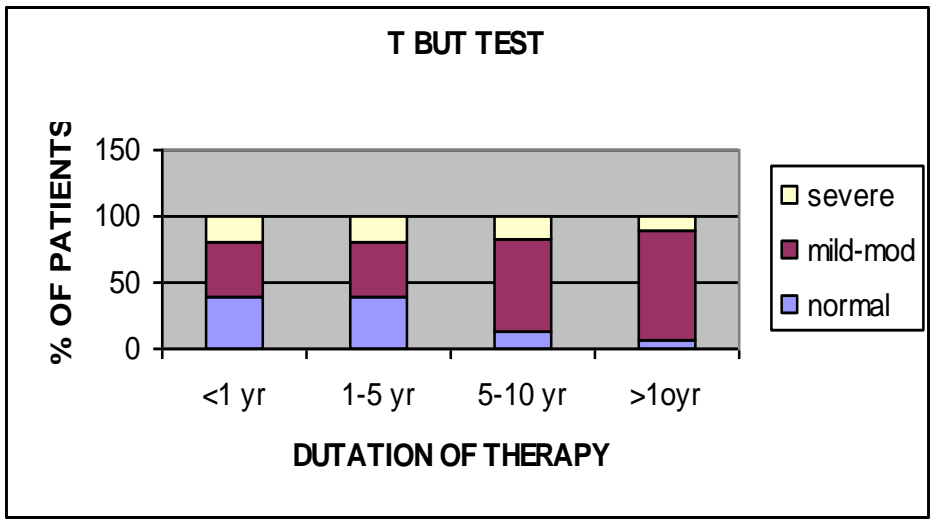

2C

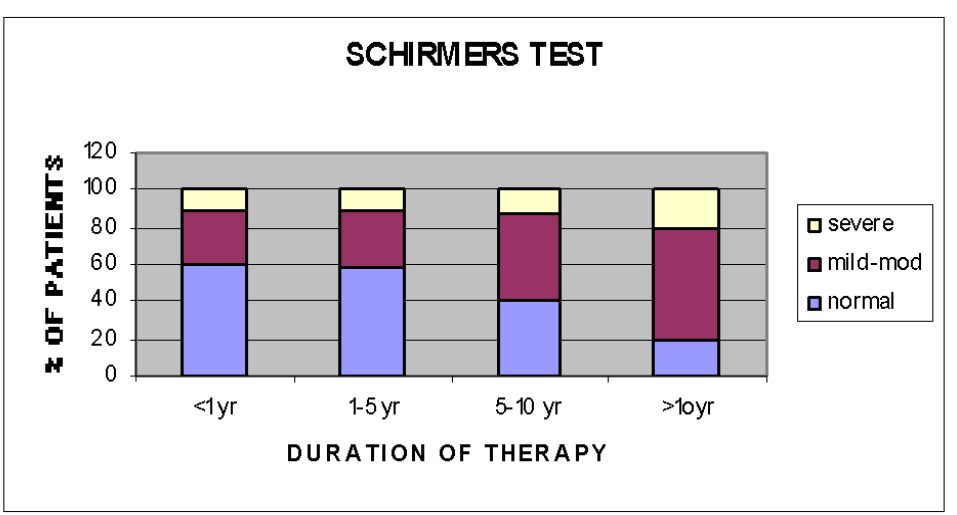

2B

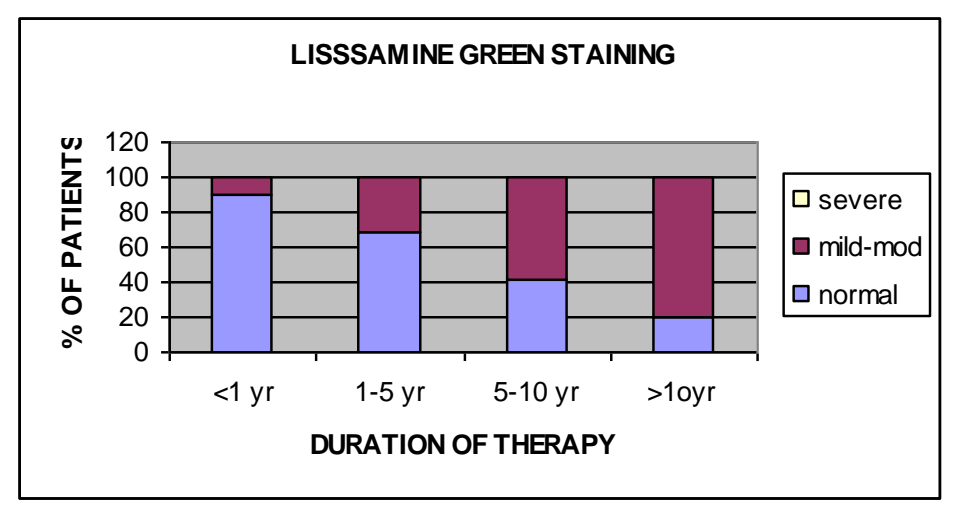

2D 